\title{
Eine weitere agglutinatorische Untersuchung von Paradysenteriebazillen.
}

\author{
Von \\ Prof. Dr. Kaoru Aoki. \\ (青木 薁) \\ (Aus dein bakteriologischen Institute der Universitüt zu Sendai.)
}

In der früheren Mitteilung haben wir bemerlit, dass es von Natur unmöglich sei, die Dysenteriebazillen übereinstimmend nach ihren zwei Eigenschaften, nämlich der agghtinierenden und fermentativ wirkenden in exakte Unterarten einzuteilen, obwohl sich schon viele Forscher darum bemüht haben. Dasselve haben auch schon Braun und Liess bemerkt. Deshalb ist es nicht nur logischer, sondern auch zweckmässiger, diese Miikroben nach ihren einzelnen Eigenschaften zu bestimmen. Von dieser Anschaunng ansgehend, habe ich sie durch gekreuzte Agglutination genan untersucht. Es stellte sich dabei heraus, dass sie deutlich in acht Unterarten teilbar sind, von denen 4 Unterarten, nämlich die erste, sechste, siebente und achte, nicht nur gegenseitig, sondern anch gegen die übrigen Unterarten stark differenziert sind, so dass man sie ganz leicht voneinander unterscheiden kann. Die vier übrigen Unterarten, nämlich die zweite, dritte, vierte und fünfte, sind diejenigen, welche manchmal gegenseitig sehr stark reagieren, so dass es zuweilen schwer fällt, sie voneinander zu unterscheiden. Wenn man aber die verschiedenen komplizierten agglutinatorischen Beziehungen zwischen den einzelnen Unterarten genau berücksichtigt, so sind sie doch deutlich voneinander zu unterscheiden.

Als ich auf diese Weise die Dysenteriebazillen agglutinatorisch in acht Unterarten eingeteilt hatte, wurde ferner untersucht, in was für einer Beziehung unsere Unterarten zu den anderen stehen, welche von verschiedenen Autoren angegeben worden sind. $\mathrm{Zu}$ diesem $\mathrm{Zwecke}$ wurden die unten bezeichneten Unterarten von Dy'senteriebazillen von verschiedenen Seiten bezogen: 1. Sieben Stämme Flexnerbazillen, 
fünf Stämme Y-bazillen, ein Stamın Strong bazillen. Diese drei Unterarten sind wach der Lentz'schen Einteilung bestimmt worden. 2. Sechs Stämme II. Typus von Shiga; vier Stämme III. Typus von Shiga; sechs Stämme IV. Typus von Shiga; drei Stämme V. Typus von Shiga, welche alle nach der Shiga'schen Einteilung eingeteilt worden sind. 3. Vier Stämme Komagome A und sechs Stämme Komagome B, welche nach der Futaki'schen Einteilung bestimmt worden sind. Diese drei Einteilungen sind die, welche in Japan gewöhnlich gebraucht werden. Dazu wurden auch viele Stämme Shigabazillen von verschiedenen Seiten bezogen.

Zuerst wurden sie mikroskopisch und kulturell genau untersucht, ob sie alle dieselben Eigenschaften aufweisen können, wie ich das in einer früheren Mitteilung bemerkt habe. Aber ihre besondere fermentative Eigenschaft, durch die man Dysenteriebazillen in verschiedene Typen einzuteilen pflegt, wurde hier nicht besonders untersucht aus dem von mir oben hervorgehobenen Grunde. Deshalb wurden sie zuerst mit acht Sera, welche unsere acht Typen Dysenteriebazillen repräsentieren, nämlich Dy. I, II, III, IV, V, VI, VII, VIII, agglutinatorisch genall untersucht. Es ergab sich, dass fast alle Stämme in den ersten fünf Unterarten bestimmt werden können. Neun Stämme wurden nämlich als zu der ersten Unterart; fünf Stämme als zu der zweiten; fünf Stämme als zu der dritten Unterart; sechs Stämme als zu der vierten Unterart; zehn Stämme als zu der fünften Unterart gehörig festgestellt. Hier muss bemerkt werden, dạss die Stämme, welche als entweder zu der dritten oder zu der fünften Unterart gehörig angenommen worden waren, manchmal schwer voneinander unterscheidbar waren. Ferner muss bemerkt werden, dass die Stämme, welche als zu der dritten Unterart gehörig festgestellt worden waren, im Serum von Shiga bazillen sehr stark agglutinierten.

Wenn man diese Einteilung mit den anderen, welche von den verschiedenen Autoren, wie oben bemerkt, angegeben worden sind, genau vergleicht, so ergibt sich folgendes: Neun Stämme, welche als zu der ersten Unterart gehörig angenommen worden sind, enthalten Stämme aus den folgenden Typen: Flexner, Y, II. u. III. 'Iypus von Shiga, und Komagome B. Fünf Stämme, welche als zur zweiten Unterart gehörig festgestellt worden sind, enthalten Stämme aus den folgenden Typen: Flexner, Y, IV. u. V. Typus von Shiga; fünf Stämme, welche als zur dritten Unterart gehörig bestimmt worden sind, umfassen Stämme aus den folgenden Typen: Flexner, II, IV u. V 
von Shiga. Sechs Stämme, welche als zu der vierten Unterart gehörig angenommen worden sind, umfassen die Stämme aus den folgenden Typen : Y, Komagome A u. B. Zehn Stämme, welche als zu der fünften gehörig festgestellt sind, umfassen die Stämme aus den folgenden Typen: Flexner, Y, II, III u. IV von Shiga und Komagome B. Wenn dieses Resultat umgekehrt von der anderen Seite betrachtet wird, kann man es auch folgendermassen ausdrücken: Alle vier Stämme Komagome A wurden nur in einer Unterart, nämlich in der vierten Unterart, gefunden, wie ich schon in einer anderen Mitteilung bemerkt habe. Sechs Stämme Komagome B wurden dagegen auf verschiedene Unterarten verteilt gefunden, d.h. anf drei Unterarten und zwar auf die erste, vierte und fünfte, in einer früheren Untersuchung auf vier Unterarten. Ebenso verhielten sich die Unterarten von Lentz, so dass die Stämme vom Flexner Typus in der ersten, zweiten, dritten und fünften; die Stämme vom Y-Typus in der ersten, zweiten, vierten und fünften Unterart verteilt nachgewiesen wurden. Ebenfalls wurden die Stämme, welche einzelne Typen von Shiga darstellen, nicht in einer Unterart, sondern in verschiedenen Unterarten verteilt gefunden. So wurden die Stämme von II Typus von Shiga in der ersten, dritten und fünften; die Stämme von III Typus von Shiga in der ersten und fünften ; die Stämme von IV Typus von Shiga in der zweiten, dritten und fünften; die Stämme von V Typus von Shiga in der zweiten, dritten und fünften Unterart verteilt nachgewiesen. Nach diesen Resultaten kann man allerdings annehmen, dass die Typen, welche von verschiedenen Autoren, wie Lentz, Shiga und Futaki angegeben wurden, agglutinatorisch nicht einheitlich betrachtet worden sind. Da aber bei dieser Untersuchung die Bakterien nur ron einer Seite aus, nämlich von ihrer agglutinierenden Eigenschaft betrachtet worden sind, wurden sie ferner auf ihre zwei Eigenschaften hin, nämlich die agglutinierende und Agglutinin bildende, genau untersucht, weil man nur auf diese Weise die agglutinatorische Identität der Bakterienstämme vollkommen feststellen kann, wie ich immer betont habe. $\mathrm{Zn}$ diesem Zwecke wurden sämtliche Stämme Dysenteriebazillen, welche bei obiger Untersuchung in unseren acht Sera agglutinatorisch geprüft und in unsere fünf Unterarten eingeteilt worden sind, Kaninchen eingespritzt auf die Weise, womit wir Kaninchen vorzubehandeln pflegen, um wirksame agglutinierende Sera herzustellen. Dadurch war ich imstande, den sämtlichen Stämmen entsprechende agglutinatorische Sera herzustellen. Mit diesen Sera wurde die gekrenzte Agglutination ausgeführt 
unter den Stämmen, welche unsere einzelnen Unterarten darstellen, anderseits unter den Stämmen, welche einzelne Typen darstellen, welche von verschiedenen Forschern angegeben worden sind. Die Resultate wurden in zwei Tabellen, nämlich in der ersten und zweiten, genau dargestellt. Aus Tabelle 1 kann man leicht ersehen, dass die Stämme, welche in der ersten Untersuchung zu einer und derselben Unterart gehörig genommen worden sind, gegenseitig gleich stark bis zum Titer agglutinieren können, nämlich sich agghtutinierend und Agglutinin bildend gleich verhalten (Tab. I). Dagegen sieht man aus der Tabelle 2, dass die Stämme, welche z.B. den Len tz'schen Typen angehören, wie dem Flexner- und Y-Typus, gegenseitig nicht immer gleich stark agglutinieren können. Ebenso verhalten sich die Stämme, welche entweder zu den Shiga'schen Typen oder Futaki'schen Typen gehören sollen. Aber es muss bemerkt werden, dass die Stämme, welche als zu Komagome A gehörig angenommen worden sind, ausnahmsweise gegenseitig gleich stark agglutinieren können, so dass man annehmen muss, dass dieser Typus allein agglutinatorisch einbeitlich ist. Diese Erfahrung wurde schon in den früheren Mitteilung gemacht (Tab. II). Aus diesen Ergebnissen ist sicher erwiesen, dass sich unsere Einteilung der Dysenteriebazillen agglutinatorisch ganz einheitlich verhält, dagegen die anderen, welche von Lentz, Shiga und Futaki vorgeschlagen worden sind, nicht, wie schon viele Forscher bemerkt haben (Krägel, Kesava Pai u. Rama Krishan, Lunz, Morgan, Ohno, Romm u. Balaschow, Schroeter u. Gutjahr u.a.). Infolgedessen beantwortet sich, wie ich schon in der früheren Mitteilung bemerkt habe, die Frage von selbst: Weshalb wurden von verschiedenen Autoren nicht selten solche Stämme unter den Flexnerbazillen nacbgewiesen, welche im Flexnerserum ganz schwach oder gar nicht reagieren können? Dieselbe Erscheinung wurde auch bei Y-Stämmen nachgewiesen. Falls der Flex nertypus oder Y-Typus agglutinatorisch einheitlich dargestellt worden wäre, wäre es ganz undenkbar gewesen, diese Erscheinung zu beobachten. Natürlich dürfen in diesem Falle die Agglutinabilität der Stämme und die Wirlssamkeit der Immunsera nicht beeinträchtigt sein. Noch einige wenige Stämme Dysenteriebazillen, welche bei obigen Untersuchungen entweder gar nicht oder nicht dentlich bestimmt werden konnten, wurden hier eingehend auf ihre zwei Eigenschaften, nämlich die agglutinierende und Agglutinin bildende hin, untersucht. Es waren im ganzen 7 Stämme. Dabei wurde festgestellt, dass der Stamm Dy. 167, welcher als der Typus von Strong, und ein anderer 
a (Dy. I).

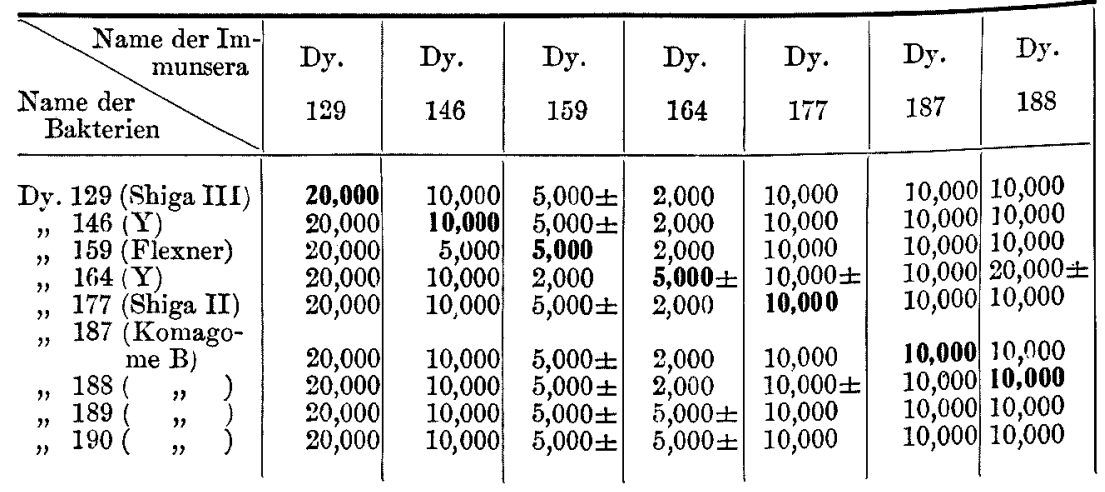

b (Dy. II).



\begin{tabular}{|c|c|c|c|c}
\hline $\begin{array}{c}\text { Dy. } \\
126\end{array}$ & $\begin{array}{c}\text { Dy. } \\
131\end{array}$ & $\begin{array}{c}\text { Dy. } \\
134\end{array}$ & $\begin{array}{c}\text { Dy. } \\
174\end{array}$ & $\begin{array}{c}\text { Dy. } \\
173\end{array}$ \\
\hline $\mathbf{2 , 0 0 0}$ & $1,000 \pm$ & 5,000 & 5,000 & $10,000 \pm$ \\
2,000 & 2,000 & 10,000 & 10,000 & 10,000 \\
2,000 & $2,000 \pm$ & $\mathbf{1 0 , 0 0 0}$ & 10,000 & 10,000 \\
2,000 & 1,000 & $10,000 \pm$ & $\mathbf{1 0 , 0 0 0}$ & 10,000 \\
2,000 & $\mathbf{2 , 0 0 0 \pm}$ & $10,000 \pm$ & 10,000 & $\mathbf{1 0 , 0 0 0}$
\end{tabular}

c (Dy. III).

\begin{tabular}{|c|c|c|c|c|c|}
\hline $\begin{array}{l}\text { Name der Im- } \\
\text { munsera } \\
\text { Name der } \\
\text { Bakterien }\end{array}$ & $\begin{array}{l}\text { Dy. } \\
124\end{array}$ & $\begin{array}{l}\text { Dy. } \\
127\end{array}$ & $\begin{array}{l}\text { Dy. } \\
168\end{array}$ & $\begin{array}{l}\text { Dy. } \\
169\end{array}$ & $\begin{array}{l}\text { Dy. } \\
171\end{array}$ \\
\hline $\begin{array}{c}\text { Dy. } 124 \text { (Shiga II) } \\
\text { " } 127 \text { (Shiga V) } \\
" 168 \text { (Flexner) } \\
" 169 \text { (Shiga II) } \\
" 171 \text { (Shiga IV) }\end{array}$ & $\begin{array}{l}20,000 \\
20,000 \\
20,000 \\
20,000 \\
20,000\end{array}$ & $\begin{array}{r}10,000 \\
\mathbf{2 0 , 0 0 0} \\
10,000 \\
5,000 \\
10,000\end{array}$ & $\begin{array}{l}2,000 \\
2,000 \\
2,000 \\
2,000 \\
2,000\end{array}$ & $\begin{array}{l}10,000 \\
10,000 \\
10,000 \\
10,000 \\
10,000\end{array}$ & $\begin{array}{l}20,000 \\
20,000 \\
20,000 \\
20,000 \\
\mathbf{2 0 , 0 0 0}\end{array}$ \\
\hline
\end{tabular}



Dy. 119 (Flexner)

, 121 (Shiga II)

" 125 (Shiga III)

, 133 (Flexner)

"145 (,")

" 154 (Shiga IV)

" 356 (Shiga III)

" 165 (Shiga II)

" 175 (Y)

" 192 (Komagome B)

Stamm Dy. 122, welcher als IV. Typus von Shiga bezeichnet war, eine vou den anderen ganz gesonderte Stelle einnimmt, weil diese beiden von den anderen Sera, welche unsere einzelnen Unterarten darstellen, fast gar nicht beeinflusst wurden. Das Serum, welches mit dem vorderen Stamme, nämlich Dy. 167, hergestellt war, konnte nämlich alle anderen Stämme, welche alle anderen Unterarten darstellen, nicht beeinflussen. Das andere Serum, welches mit letzterem Stamme, nämlich Dy. 122, 
I.

\begin{tabular}{r|c}
\hline Dy. & Dy. \\
189 & 190 \\
\hline 10,000 & 10,000 \\
10,000 & 10,000 \\
10,000 & 10,000 \\
10,000 & $10,000 \pm$ \\
10,000 & 10,000 \\
10,000 & 10,000 \\
5,000 & $10,000 \pm$ \\
$\mathbf{1 0 , 0 0 0}$ & $\mathbf{1 0 , 0 0 0}$ \\
10,000 & $\mathbf{1 0 , 0 0 0}$
\end{tabular}

d (Dy. IV).



e (Dy. V).

\begin{tabular}{|c|c|c|c|c|c|c|c|c|c|}
\hline Dy. & Dy. & $\mathrm{D}_{5}$. & Dy. & Dy. & Dy. & Dy. & Dy. & Dy. & Dy. \\
\hline 119 & 121 & 125 & 133 & 145 & 154 & 156 & 165 & 175 & 192 \\
\hline 5,000 & 1,000 & 10,000 & 5,000 & 10,000 & 2,000 & 2,000 & 2,000 & 10,000 & $10,000 \pm$ \\
\hline $10,000 \pm$ & 2,000 & $10,000 \pm$ & 10,000 & 5,000 & $2,000 \pm$ & $2,000 \pm$ & 2,000 & $10,000 \pm$ & 10,000 \\
\hline 10,000 & $2,000 \pm$ & 10,000 & 10,000 & 10,000 & $5,000 \pm$ & $5,000 \pm$ & 5,000 & 10,000 & 10,000 \\
\hline 10,000 & 2,000 & 10,000 & 10,000 & 10,000 & $5,000 \pm$ & 2,000 & 5,000 & 10,000 & $10,000 \pm$ \\
\hline $10,000 \pm$ & 2,000 & 10,000 & 10,000 土 & 10,000 & 5,000 & 2,000 & 2,000 & 10,000 & 10,000 \\
\hline 10,000 & 1,000 & 10,000 & $10,000 \pm$ & 10,000 & 5,000 & 5,000 & 2,000 & 10,000 & $10,000 \pm$ \\
\hline $10,000 \pm$ & $2,000 \pm$ & 10,000 & 10,000 & 10,000 & $5,000 \pm$ & 5,000 & 2,000 & 10,000 & $10,000 \pm$ \\
\hline 10,000 & 2,000 & 10,000 & 10,000 & 10,000 & 5,000 & 5,000 & 2,000 & 10,000 & 10,000 \\
\hline 10,000 & $2,000 \pm$ & 10,000 & 10,000 & 10,000 & $5,000 \pm$ & $5,000 \pm$ & 5,000 & 10,000 & 10,000 \\
\hline 10,000 & 2,000 & 10,000 & 10,000 & 10,000 & 5,000 & 5,000 & 5,000 & 10,000 & 10,000 \\
\hline
\end{tabular}

hergestellt worden war, agglutinierte alle anderen acht Unterarten vertretenden Stämme, obwohl einige Stämme von ihnen ziemlich stark beeinflusst wurden, doch viel schwächer als der Titer. Dazu agglutinierten die beiden Stämme gegenseitig nicht. Deshalb muss man annehmen, dass die beiden Stämme einzeln eine selbständige neue Unterart darstellen. Der vordere Stamm stellt die neunte Unterart, der letztere die zehnte Unterart dar. Der noch übrige Stamm Dy. 138 muss als zu der fünften Unterart gehörig angenommen werden, weil er nicht nur mit dem Stamme aus der fünften Unterart gegenseitig gleich stark, sondern auch in den anderen Sera, welche andere Unterarten darstellen, immer im gleichen Verhalten wie der Stamm aus der fünften Unterart, reagieren konnte. Die vier übrigen Stämme, welche einmal zu der dritten Unterart, ein andermal zu der fünften Unterart eine nahe 
K. Aok i

Tabelle

a (Flexner).

\begin{tabular}{|c|c|c|c|c|c|c|}
\hline Name der Im- & $\begin{array}{l}\text { Dy. } \\
119\end{array}$ & $\begin{array}{l}\text { Dy. } \\
133\end{array}$ & $\begin{array}{l}\text { Dy. } \\
145\end{array}$ & $\begin{array}{l}\text { Dy. } \\
159\end{array}$ & $\begin{array}{l}\text { Dy. } \\
168\end{array}$ & $\begin{array}{l}\text { Dy. } \\
174\end{array}$ \\
\hline $\begin{array}{l}\text { Dy. } 119 \text { (Dy. V) } \\
" 133 \text { (Dy. V) } \\
" 145 \text { (Dy. V) } \\
" 159 \text { (Dy. I) } \\
" 168 \text { (Dy. III) } \\
" 174 \text { (Dy. II) }\end{array}$ & $\begin{array}{c}\mathbf{5 , 0 0 0} \\
10,000 \\
10,000 \pm \\
100 \pm \\
20,000 \pm \\
2,000\end{array}$ & $\begin{array}{c}5,000 \\
10,000 \\
10,000 \pm \\
10,000 \pm \\
20,000 \\
5,000\end{array}$ & $\begin{array}{c}10,000 \\
10,000 \\
10,000 \\
1,000 \pm \\
20,000 \\
2,000\end{array}$ & $\begin{array}{c}500 \\
1,000 \pm \\
200 \pm \\
10,000 \\
1,000 \\
100\end{array}$ & $\begin{array}{l}\mathbf{2}, 000 \\
5,000 \\
5,000 \\
100 \pm \\
\mathbf{5 , 0 0 0} \\
\mathbf{5 , 0 0 0}\end{array}$ & $\begin{array}{c}500 \pm \\
1,000 \\
200 \pm \\
100- \\
1,000 \\
10,000\end{array}$ \\
\hline
\end{tabular}

b (Y).

\begin{tabular}{|c|c|c|c|c|c|}
\hline $\begin{array}{c}\text { Name der Im- } \\
\text { munsera } \\
\text { Name der } \\
\text { Bakterien }\end{array}$ & $\begin{array}{l}\text { Dy. } \\
120\end{array}$ & $\begin{array}{l}\text { Dy. } \\
134\end{array}$ & $\begin{array}{l}\text { Dy. } \\
146\end{array}$ & $\begin{array}{l}\text { Dy. } \\
164\end{array}$ & $\begin{array}{l}\text { Dy. } \\
175\end{array}$ \\
\hline $\begin{array}{c}\text { Dy. } 120 \text { (Dy. IV) } \\
, 134 \text { (Dy. II) } \\
" 146 \text { (Dy. I) } \\
" 164 \text { (Dy. I) } \\
, 175 \text { (Dy. V) }\end{array}$ & $\begin{array}{c}\mathbf{1 0 , 0 0 0} \\
2,000 \\
100 \\
200 \\
5,000 \pm\end{array}$ & $\begin{array}{c}100 \\
10,000 \\
100- \\
1,000 \pm \\
2,000\end{array}$ & $\begin{array}{r}200 \\
500 \\
10,000 \\
5,000 \\
1,000\end{array}$ & $\begin{aligned} & 100 \\
& 500 \\
& \mathbf{2}, 000 \\
& \mathbf{5 , 0 0 0} \\
& \mathbf{2}, 000\end{aligned}$ & $\begin{array}{c}5,000 \\
10,000 \\
2,000 \\
100 \pm \\
10,000\end{array}$ \\
\hline
\end{tabular}

c (Shig a II).

\begin{tabular}{|c|c|c|c|c|c|}
\hline $\begin{array}{l}\text { Name der Im- } \\
\text { munsera } \\
\text { Name der } \\
\text { Bakterien }\end{array}$ & $\begin{array}{l}\text { Dy. } \\
121\end{array}$ & $\begin{array}{l}\text { Dy. } \\
124\end{array}$ & $\begin{array}{l}\text { Dy. } \\
165\end{array}$ & $\begin{array}{l}\text { Dy. } \\
169\end{array}$ & $\begin{array}{l}\text { Dy. } \\
177\end{array}$ \\
\hline $\begin{array}{c}\text { Dy. } 121 \text { (Dy. V) } \\
\quad, 124 \text { (Dy. III) } \\
\Rightarrow 165 \text { (Dy. V) } \\
\Rightarrow 169 \text { (Dy. III) } \\
\Rightarrow 177 \text { (Dy. I) }\end{array}$ & $\begin{array}{l}2,000 \\
2,000 \\
2,000 \\
5,000 \\
1,000\end{array}$ & $\begin{array}{l}10,000 \\
\mathbf{2 0 , 0 0 0} \\
20,000 \\
20,000 \\
10,000\end{array}$ & $\begin{array}{l}10,000 \pm \\
10,000 \\
10,000 \\
30,000 \\
2,000\end{array}$ & $\begin{array}{c}5,000 \pm \\
10,000 \\
10,000 \pm \\
10,000 \\
2,000\end{array}$ & $\begin{array}{r}5,000 \\
5,000 \\
5,000 \\
5,000 \\
10,000\end{array}$ \\
\hline
\end{tabular}

d (Shig a III).

\begin{tabular}{l|c|c|c}
\hline $\begin{array}{c}\text { Name der Im- } \\
\text { munsera }\end{array}$ & Dy. & Dy. & Dy. \\
$\begin{array}{c}\text { Name der } \\
\text { Bakterien }\end{array}$ & 125 & 129 & 156 \\
\hline Dy. 125 (Dy. V) & $\mathbf{1 0 , 0 0 0}$ & $\mathbf{5 , 0 0 0}$ & $5,000 \pm$ \\
"129 (Dy. I) & 1,000 & $\mathbf{2 0 , 0 0 0}$ & 500 \\
$" 156$ (Dy. V) & 10,000 & $5,000 \pm$ & $\mathbf{5 , 0 0 0}$
\end{tabular}


2.

e (Shiga IV).

\begin{tabular}{l|l|l|l|r}
\hline $\begin{array}{c}\text { Name der } \\
\text { Immunsera }\end{array}$ & Dy. & Dy. & Dy. & Dy. \\
$\begin{array}{c}\text { Name der } \\
\text { Bakterien }\end{array}$ & 126 & 154 & 171 & 178 \\
\hline Dy. 126 (Dy. II) & $\mathbf{2 , 0 0 0}$ & $\mathbf{2 , 0 0 0 \pm}$ & 20,000 & 10,000 \\
"154 (Dy. V) & 500 & $\mathbf{5 , 0 0 0}$ & 5,000 & 5,000 \\
"171 (Dy. III) & 1,000 & $\mathbf{2 , 0 0 0}$ & $\mathbf{2 0 , 0 0 0}$ & $\mathbf{2 0 , 0 0 0}$ \\
"178 (Dy. II) & $\mathbf{2 , 0 0 0}$ & 5,000 & $\mathbf{2 0 , 0 0 0}$ & $\mathbf{1 0 , 0 0 0}$ \\
& & & &
\end{tabular}

f (Shiga V).

\begin{tabular}{|c|c|c|c|}
\hline Name der & $\begin{array}{l}\text { Dy. } \\
127\end{array}$ & $\begin{array}{l}\text { Dy. } \\
131\end{array}$ & $\begin{array}{l}\text { Dy. } \\
157\end{array}$ \\
\hline $\begin{array}{c}\text { Dy. } 127 \text { (Dy. (III) } \\
", 131 \text { (Dy. II) } \\
, 157 \text { (Dy. V) }\end{array}$ & $\begin{array}{c}\mathbf{1 0 , 0 0 0} \\
5,000 \\
1,000 \pm\end{array}$ & $\begin{array}{r}5,000 \\
2,000 \\
500\end{array}$ & $\begin{array}{l}5,000 \\
2,000 \\
\mathbf{5 , 0 0 0}\end{array}$ \\
\hline
\end{tabular}

g (Komagome A).

\begin{tabular}{l|l|l|l|l}
\hline $\begin{array}{c}\text { Name der } \\
\text { Immunsera }\end{array}$ & Dy. & Dy. & Dy. & Dy. \\
$\begin{array}{c}\text { Name der } \\
\text { Bakterien }\end{array}$ & 135 & 160 & 185 & 186 \\
\hline Dy. 135 (Dy. IV) & $\mathbf{1 0 , 0 0 0}$ & 10,000 & 10,000 & 10,000 \\
"160 (Dy. IV) & 10,000 & $\mathbf{1 0 , 0 0 0}$ & 10,000 & 10,000 \\
"185 (Dy. IV) & 10,000 & $5,000 \pm$ & $\mathbf{1 0 , 0 0 0}$ & 10,000 \\
"186 (Dy. IV) & 10,000 & $5,000 \pm$ & 10,000 & $\mathbf{1 0 , 0 0 0}$
\end{tabular}

h (Komagome B).

\begin{tabular}{|c|c|c|c|c|c|c|}
\hline $\begin{array}{c}\text { Name der Im } \\
\text { munsera } \\
\text { Name der } \\
\text { Bakterien }\end{array}$ & $\begin{array}{l}\text { Dy. } \\
161\end{array}$ & $\begin{array}{l}\text { Dy. } \\
187\end{array}$ & $\begin{array}{l}\text { Dy. } \\
188\end{array}$ & $\begin{array}{l}\text { Dy. } \\
189\end{array}$ & $\begin{array}{l}\text { Dy. } \\
190\end{array}$ & $\begin{array}{l}\text { Dy. } \\
192\end{array}$ \\
\hline $\begin{array}{c}\text { Dy. } 161 \text { (Dy. IV) } \\
\text { " } 187 \text { (Dy. I) } \\
\text { " } 188 \text { (Dy. I) } \\
" 189 \text { (Dy. I) } \\
" 190 \text { (Dy. I } \\
" 192 \text { (Dy. V) }\end{array}$ & $\begin{array}{c}10,000 \\
1,000 \pm \\
500 \\
2,000 \\
1,000 \pm \\
5,000 \pm\end{array}$ & $\begin{array}{c}1,000 \pm \\
10,000 \\
10,000 \\
10,000 \\
10,000 \\
5,000\end{array}$ & $\begin{array}{c}5,000 \\
10,000 \\
10,000 \\
10,000 \\
10,000 \\
20,000 \pm\end{array}$ & $\begin{array}{r}1,000 \\
10,000 \\
5,000 \\
10,000 \\
10,000 \\
2,000\end{array}$ & $\begin{array}{c}2,000 \pm \\
10,000 \\
10,000 \pm \\
10,000 \\
\mathbf{1 0 , 0 0 0} \\
\mathbf{2}, 000\end{array}$ & $\begin{array}{r}5,000 \\
5,000 \\
500 \\
500 \\
500 \\
10,000\end{array}$ \\
\hline
\end{tabular}


Beziehung zeigten, konnten bei genauerer Untersuchung als zu irgend einer Unterart gehörig nicht bestimmt werden. Doch wurden sie nicht nur gegenseitig, sondern auch gegenüber den anderen Unterarten nicht so scharf differenziert, so dass sie keine neuen selbständigen Unterarten darstellen können. Deshalb muss man annehmen, dass sie verschiedene Zwischenformen darstellen. Diese Zwischenformen dürften dadurch zustande gekommen sein, dass Paradysenteriebazillen immer geneigt sind, verschiedene Variationen neu zu bilden. Ich habe auch darüber schon einige Erfahrungen gesammelt. Aber diese Untersuchung ist noch im Gange, so dass ich noch nicht in der Lage bin, hier näher darauf einzugehen. Allerdings würde durch dieses Verhalten auch die agglutinatorische Bestimmung von Dysenteriebazillen immer schwerer.

Ferner wurden 42 Stämme Paradysenteriebazillen, welche in unserem Institute aus Untersuchungsmaterial herausgezüchtet worden waren, in zehn Sera agglutinatorisch auf die gleiche Weise untersucht, und von ihnen stellten acht die acht Unterarten dar und die zwei übrigen die in der letzten Untersuchung neu gefundenen zwei Unterarten, nämlich die neunte und zehnte. Es ergab sich, dass die meisten Stämme zu der ersten Unterart gehörten. Es waren im ganzen 19 Stämme. Zwei Stämme wurden ferner als zu der zweiten Unterart; sechs Stämme als zu der dritten Unterart; vier Stämme als zu der vierten Unterart; sechs Stämme als zu der fünften Unterart; zwei Stämme als zu der sechsten Unterart; und noch ein Stamm als zu der zehnten Unterart gehörig festgestellt. Bei den Stämmen, welche als zu der fünften Unterart gehörig angenommen wordon waren, war es manchmal schwer, festzustellen, ob sie wirklich zu der oben genannten Unterart gehörten, weil sie manchmal so ähnlich wie die Stämme aus der dritten Unterart reagieren können. Die anderen Stämme, welche als zu den anderen übrigen Unterarten gehörig festgestellt worden waren, waren ganz leicht voneinander zu unterscheiden. Der eine von den zwei übrigen Stämmen, welche in allen zehn Sera niemals bis zum Titer, ja sogar viel schwächer als der Titer, beeinflusst worden waren, wurde als ein schwer agglutinabler Stamm, und der andere muss als eine neue Unterart betrachtet werden, weil er selbst nicht nur von den anderen Sera fast nicht agglutiniert wurde, sondern auch sein Serum alle Stämme aus anderen Unterarten nicht beeinflussen konnte. Nach diesem Ergebnisse wurde auch beobachtet, dass Paradysenteriebazillen, welche bei Dysenterie gefunden werden, meistens diejenigen Stämme sind, welche die ersten fünf Unterarten darstellen. Solche Stämme 
dagegen, welche dabei auch gefunden und die übrigen Unterarten, nämlich die sechste, siebente, neunte und zehnte, darstellen sollen, findet man ganz wenig.

Zum Schlusse wurden Shiga stämme, von welchen hier im ganzen 29 von verschiedenen Seiten gesammelt worden waren, in zehn repräsentierenden Sera agglutinatorisch untersucht. Es wurde festgestellt, dass Shiga bazillen in allen Sera bis auf Shiga serum fast nicht beeinflusst werden können. Dagegen agglutinierten sie im Shigaserum deutlich bis zum Titer. Einige Stämme, welche darin nicht reagierten, wurden als schwer agglutinable Stämme festgestellt. Wie in den anderen Mitteilungen von mir und Ogasawara, wurde auch hier festgestellt, dass, obwohl es unter den Paradysenteriebazillen solche Stämme als eine Unterart gibt, welche in Shiga serum selhr stark, ja sogar bis zum Titer agglutinieren, doch umgekehrt unter den Shig a stämmen ein solcher nicht nachzuweisen ist, welcher in Sera von Paradysenteriebazillen sehr stark beeinflusst werden kann. Ferner wurde auch hier festgestellt, dass Shiga bazillen agrglutinatorisch ganz einheitlich sind.

\section{Zusammenfassung.}

1. Stämme von Dysenteriebazillen, welche entweder nach Lentz, Shiga oder nach Futaki in verschiedene Typen eingeteilt worden sind, wurden meistens als zu unserer ersten fünf Unterarten gehörig nachgewiesen.

2. Darunter wurden nur diejenigen Typen als agglutinatorisch einheitlich festgesellt, welche Shigabazillen und Komagome A von Futaki darstellen. Die übrigen Typen wurden als agglutinatorisch nicht einheitlich nachgewiesen.

3. Shiga bazillen wurden nämlich als mit unserer achten; Komagome A als unit unserer vierten Unterart identisch festgestellt. Stämme aus den übrigen Typen wurden teils als zu der ersten, teils zu der zweiten, teils zu der dritten, teils zu der fünften Unterart gehörig nachgewiesen.

4. Paradysenteriebazillen, welche bei Dysenteriekranken gefunden wurden, sind diejenigen, welche unsere ersten fünf Unterarten darstellen. Unsere anderen zwei Unterarten sind sehr selten nachzuweisen.

5. Bei dieser Untersucbung wurden ferner ausser den unsere acht Unterarten darstellten Stämmen noch drei Stämme nachgewiesen, welche einzeln verschiedeñe nene Unterarten darstellen, weil sie einer- 
seits in den anderen die acht Unterarten vertretenden Sera fast nicht reagieren, anderseits ihre Sera alle acht Stämme, welche diese acht Unterarten repräsentieren, nicht agglutinieren konnten. Sie wurden die neunte, zehnte und elfte Unterart genannt.

\section{Literatur.}

(1) A oki, Über die agglutinatorische Einteilnng von Dysenteriebazillen, Tohoku Journ. of Exp. Med., 1921, 2, 142.

(2) Brau n u. Liess, UUber Colitisbazillen, Ztschr. f. Hyg., 1919, 88, 251.

(3) Brau n, Ätiologie der bazillären Ruhr, Ztschr. f. kl. Med., 1921, 91, 304.

(4) Krägel, Über die Ruhragglutinine, insbesondere ihr Verhalten in Krankenseren, Ctrlbl. f. Bakt., 1 Abt. Orig., 1911, 58, 48.

(5) Kesava Pai u. Rama Krishan, The differentiation of dysentery bacilli by their agglutination reaction, Indian Journ. of Med. Research, 1916, 3, 763.

(6) Lu z, Über die Erreger der bazillären Dysenterie, Ctrlbl. f. Bakt., 1 Abt. Orig., $1910,56,28$.

(7) Liess, Über Colitisbazillen, Ctrlbl. f. Bakt., 1 Abt. Orig., 1919, 83, 193.

(8) Morgan, The Differentiation of the mannit fermenting group of bacillus dysenteriae, Journ. of Hyg., 1911, 11, 1.

(9) Ogasawara, Über Stämme Dysenteriebazillen, welche in Shiga-Serum sehr stark agglutinieren können, Tohoku Journ. of Exp. Med., 1922, 4,

(10) Ohno, The Types of bacilli of dysentery group, Philippine Journ. of Science, $1906,1,951$.

(11) Romm u. Balaschow, Über die Agglutinine in Krankenserum der Ruhrbazillen, Ctrlbl. f. Bakt., 1 Abt. Orig., 1912, 66, 426.

(12) Schroeter u. Gutjahr, Vergleichende Studien der Typhus-, Coli- und Dysenteriebakterien in Anschluss an eine kleine Ruhrepidemie in Mitteldeutschland, Ctrlbl. f. Bakt., 1 Abt. Orig. 1911, 58, 577. 